\title{
A MEMS based compact natural gas analyzer implementing IEEE-1451.2 and BS-7986 smart sensor standards
}

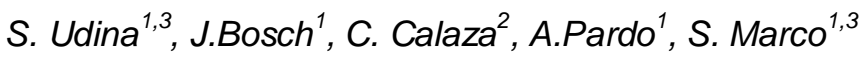 \\ ${ }^{1}$ Departament d'Electrònica, Universitat de Barcelona, Martí i Franquès, 1, 08028 Barcelona, Spain, \\ smarco@el.ub.edu \\ ${ }^{2}$ Centro Nacional de Microelectrónica-CSIC, Campus UAB, Cerdanyola, Spain \\ ${ }^{3}$ Artificial olfaction group, IBEC, Baldiri i Rexach 13, 08028 Barcelona, Spain
}

\begin{abstract}
A compact natural gas analyzer prototype is presented which incorporates several advanced features. In first place a very recently reported MEMS sensing technology for properties determination of natural gas mixtures is implemented. Besides, the prototype is a very complete smart instrument incorporating fault detection, self-identification to a network and measurement quality metrics (self validation or SEVA), provided by the combination of the IEEE-1451 and BS-7986 smart sensor standards. Experimental measurements are presented for calibration and validation of the device, with a remarkable uncertainty for the determination of Superior Calorific Value (SCV) of $1.1 \%$. The instrument demonstrates the advanced fault detection possibilities implemented and its enhanced connectivity features granted by IEEE-1451.2 The approach demonstrates the feasibility of compact, low-cost, and smart natural gas analyzers using a single MEMS microsensor and advanced smart sensor standards.
\end{abstract}

Key words: IEEE-1451, BS-7986, smart sensor, natural gas, PLS, fault detection

\begin{abstract}
Introduction
Natural gas consists mainly of Methane, with variable amounts of higher hydrocarbons, nitrogen and carbon dioxide. Its properties are strongly dependent on its origin. Precise monitoring of natural gas properties variation is of great economic and technical importance, and in particular its heating value (or calorific value), as it is a measure of the energy contained in the gas. Interest in this subject has recently been stirred after the increasing liberalization of the natural gas market in Europe.
\end{abstract}

As a consequence of this growing interest, several instruments have been proposed during the last decade, based in different physical measurements which are correlated with natural gas properties (hence the 'correlative methods' denomination) [1]. These methods complement the available previous instrumentation [2,3] and in particular the reference technology, process gas chromatography (PGC).

A new instrument has been designed, taking into consideration the stringent requirements of the particular field of natural gas analysis. Stability and resilience to a wide variety of environmental conditions is a must, and the possibility of operational self-assessment would definitely be desirable. The lack of robustness and the need of frequent recalibrations are common problems in current state-of-the.art natural gas analyzers based in PGC, implying burdensome maintenance costs. Calibration, maintenance and expert operation costs build up a considerable yearly bill, which adds up to high initial installation and acquisition costs

The presented work is directly related to a new approach recently proposed by Udina et al. $[4,5]$ which aims to perform the natural gas analysis using a single MEMS microsensor. This previous works report the sensor construction, the sensing principle and the key factors of the data analysis. The presented compact prototype implements this novel, highly cost-effective MEMS sensor approach [5], and smart sensor standards (BS-7986 and IEEE-1451) which provide other advanced features to the prototype with incidence in reliability, communications and eased maintenance.

\section{Description of the smart sensor electronics}

In order to implement the necessary firmware for IEEE-1451 and BS-7986 standards implementation, sensor excitation and reading, and signal processing, a MSP430F1612 (Texas 
Instruments inc., USA) microcontroller was selected. The device has six digital ports, two timers and an SPI port (used for the IEEE1451.2 interface) together with $55 \mathrm{~Kb}$ of Flash memory and $5 \mathrm{~Kb}$ of RAM. These characteristics provided plenty of $\mathrm{I} / \mathrm{O}$ and processing power offering good design flexibility. An external high-end analog-to-digital converter is used, the AD7711, to ensure a high quality signal acquisition due to its 24 bit (maximum) performance. Previous uncertainty analysis for the implemented sensor indicated that signal acquisition and excitation stages shall be designed very carefully to reduce its noise contribution [5]. Figure 1 shows a general block diagram of the device.

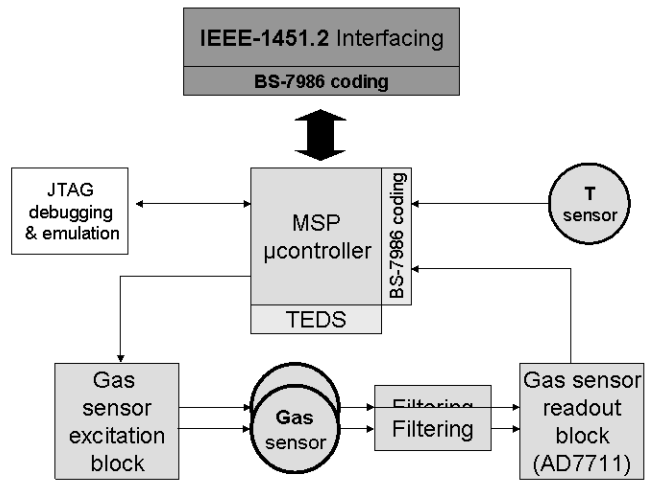

Fig. 1. prototype STIM System oveniew showing basic functional blocks as well as IEEE-1451.2 and $B S$-7986 features

\section{Combined implementation of the IEEE- 1451.2 and BS-7986 standards}

A combination of IEEE-1451 and BS-7986 standards was already proposed by Karatzas et al. in 2006 [6], as a means of rationalizing and optimizing the design of smart sensors. The herein presented implementation is basically consistent with this previous one. Figure 2 shows a block division showing the proposal in [6] together with the IEEE-1451.2 standard structure. A description of the blocks follows.

Sensor interface block: every sensor presents a firmware interface which is in charge of communicating with the sensing element hardware, obtaining measurements on demand and performing basic signal processing (and eventually units conversion) as appropriate.

Sensor model block: in [6] the Sensor Model Provider (SMP) block is in charge of providing a theoretical sensor model for a specific sensor or range of similar sensors. In effect it stores a given model data and communicates it to requesting instances. The Sensor model here presents a similar approach, the block is in charge of storing and providing a computed statistical model based on a buffer of historical data to the fault detection block. This is the first of two levels of fault detection mechanisms present in the system:

- A univariate model computed in "realtime" with statistics from a short historical measurement buffer (in this case 10 samples), which constitutes the correlation model in level one.

A multivariate model computed with a large set of historical data (the training set) based in the $Q$ residual statistics of the PLS model, which constitutes the multivariate model in level 2, note that once the model is computed offline, the model prediction and its residuals are computed in real time, the presence of unusually high values for this statistic is an indication of a faulty sensor reading [7].

Fault detection block: The Fault detection block performs the detection of faulty data in the basis of the model provided by the sensor model, quality metrics codes are assigned according to BS-7986. The fault detection block also performs sensor readings correction as proposed in [6], indicating the correction using BS-7986 codes. Moreover, this block is also in charge of estimating the uncertainty values considering the information made available to it by the sensor readings, sensor TEDS and the statistical sensor model. Compliant to the BS7986 standard, the uncertainty value denotes the error band of the associated data value at a $95 \%$ level of confidence and is represented in the same units and with the same precision as the data value. Insight on the specific algorithms for fault detection and sensor correction is provided in section 6.3.3.

Internal fusion block: The block combines the sensors' readings in one output. In this case the combination procedure is a multivariate regression model calculated using a PLS algorithm. The multivariate regression outputs the value of the superior heating value in $\mathrm{MJ} / \mathrm{m3}$. Similar regression models can be analogously obtained for several other properties [5]. 


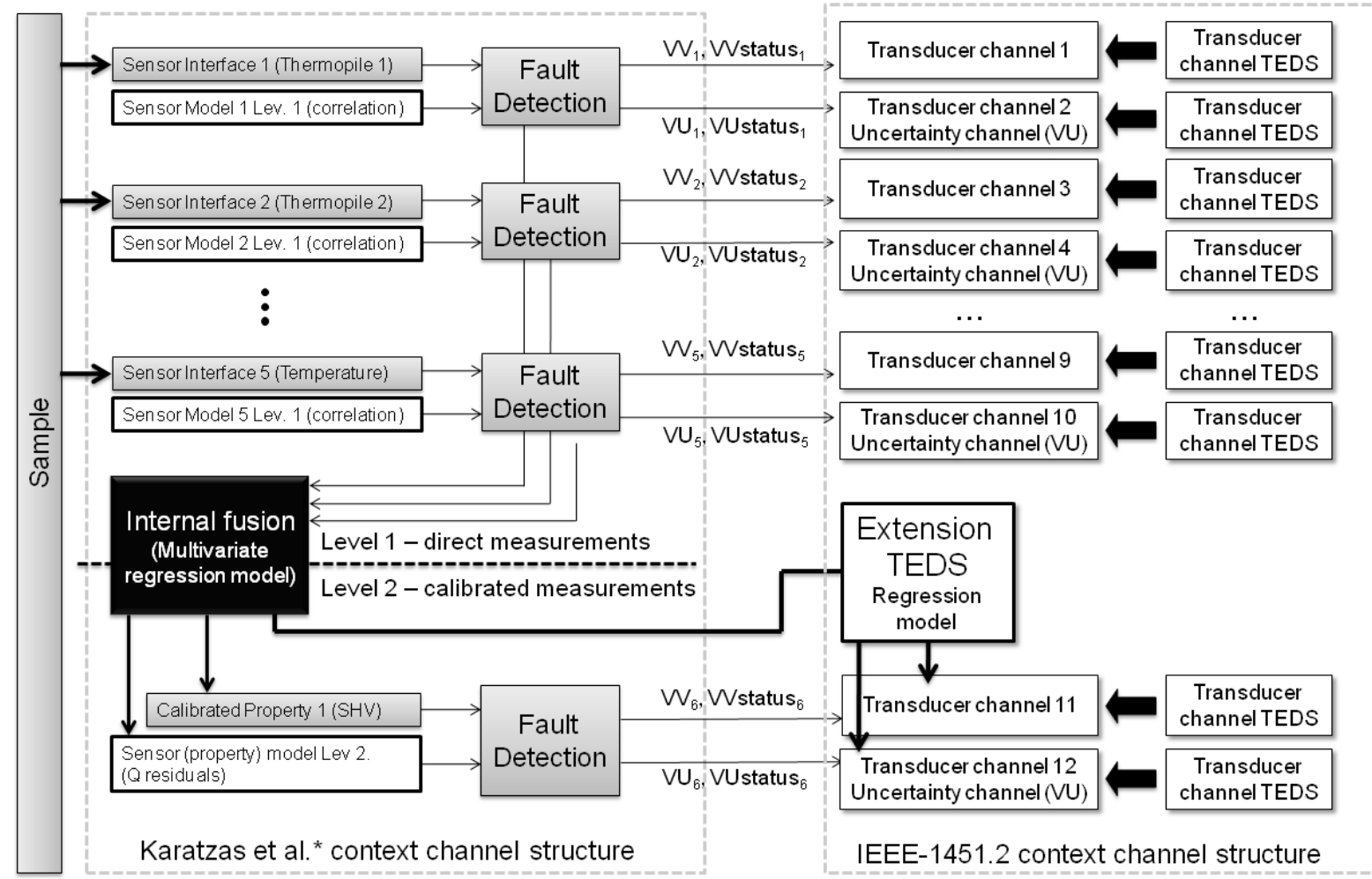

Fig. 2. IEEE-1451.2 and BS-7986 context diagram, comparison with [7] is provided.

The right side of the diagram in Fig. 2 shows the system structure regarding the IEEE-1451.2 context, once data is pre-processed, BS-7986 quality indicators added, and uncertainty calculated, the values "enter" the IEEE-1451.2 context using standard transducer channels. A more detailed description of the blocks is provided below:

Transducer channels: The IEEE-1451.2 building blocks are the transducer channels, these are instances normally associated to sensor or actuators which interact with external phenomena. However a transducer channel may also contain data regarding to internal processes or simply indirectly related to external measurements. This is the case the for Transducer channels 2, 4, 6, 8, 10, 11 and 12. Transducer channel 11 is a combination of measurements from other channels (in the current setup transducer channels 1 and 9) to obtain a calibrated property, the other channels (2, 4, 6, 8, 10 and 12) are VU (BS-7986) channels which provide a way of accessing the uncertainty estimation calculated in the fault detection block. These channels are not physical sensors, but virtual sensors according to the IEEE-1451.2 terminology. VV and VU are updated simultaneously, when a trigger for the VV channel is executed.

Transducer channel TEDS: A transducer channel is formally defined by a number of parameters and specifications, which are defined according to the IEEE-1451.2. In addition to the technical parameters in the channel TEDS, identification information is allocated in the Channel Identification TEDS. There is also specific calibration information allocated in the Calibration TEDS. Associated to them the Calibration identification TEDS provide descriptive information.

System Meta TEDS block: Global information common to all sensor channels is stored in the Meta TEDS, which contain parameter values and options. These are also complemented with a Meta-identification TEDS for system-level identification.

\section{Results}

The sensor readings were acquired using a PCsoftware emulating an IEEE-1451 host processor (NCAP).

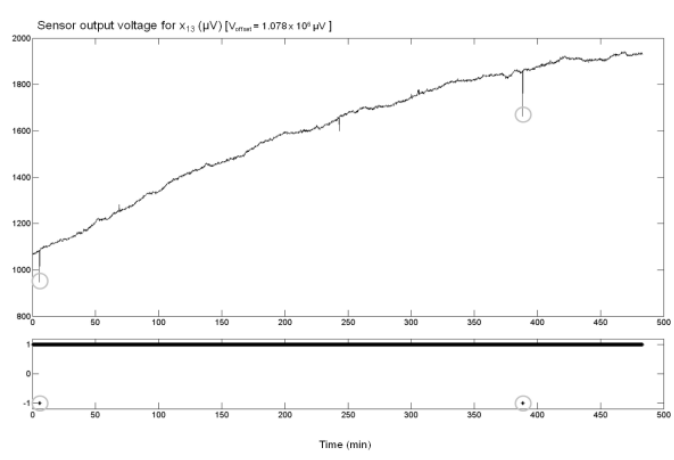

Figure 3. Example univariate fault detection 
Using this software the basic features of the sensor were tested. Figure 3 shows results for an example univariate fault detection (level 1) detecting spurious communication errors. Quality codes are assigned according to BS7986.
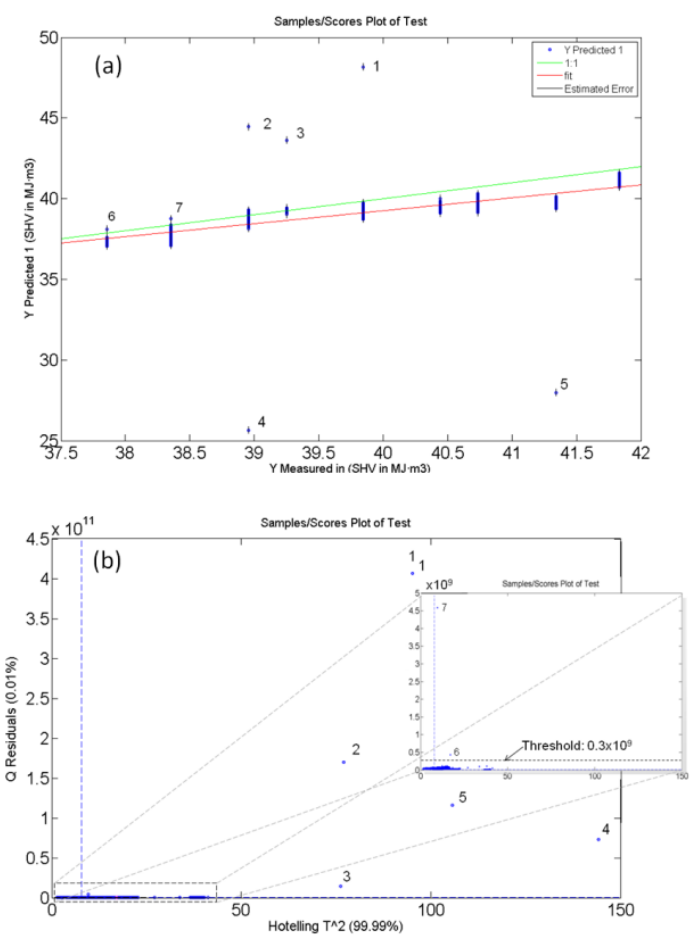

Fig. 4. Example multivariate fault detection using Q-residuals.Errors are easily detected

Figure 4 shows an example of the multivariate detection performed by the fault detection block (level 2). Again errors are correctly spotted and assigned corresponding BS-7986 quality codes. Figure 5 shows the results after sensor data correction.

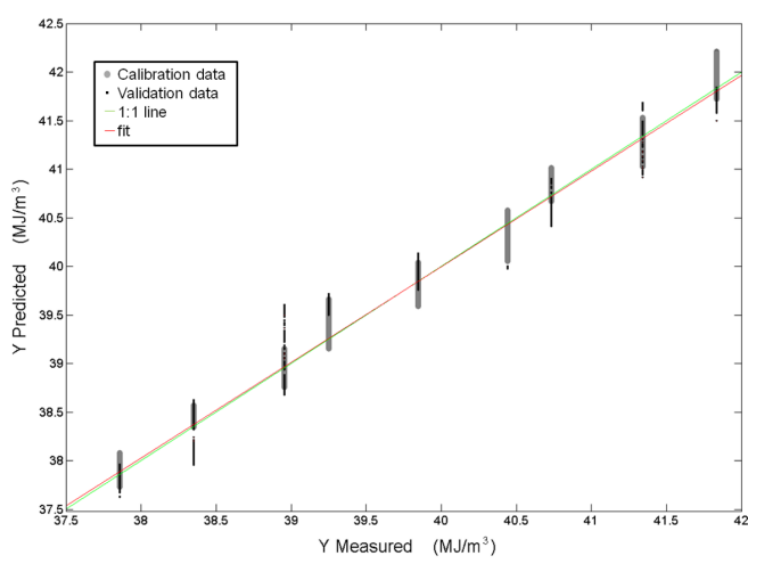

Figure 5. Calibration and validation data after sensor data correction.

Sensor data correction is based in this case on a median filter implemented with the use of historical data.

\section{Conclusions}

A prototype instrument combining IEEE-1451.2 and BS-7986 has been presented. The feasibility of the combination has been demonstrated and fault detection and correction has been shown in examples from real measurements. Besides the implementation of smart sensor standards, the prototype also is a test device for a new MEMS measurement approach based in recent developments, becoming an advanced smart sensor proposal for reliable low-cost natural gas analysis.

\section{Acknowledgements}

ISP group is a consolidated Grup de Recerca de la Generalitat de Catalunya and has support from the Departament d'Universitats, Recerca $i$ Societat de la Informació de la Generalitat de Catalunya (expedient 2009 SGR 0753). This work has received support from the Comissionat per a Universitats i Recerca del DIUE de la Generalitat de Catalunya and the European Social Fund (ESF). The investigation has been also supported by the project TEC2004-07853-C02-01.

\section{References}

[1] P. Schley, M. Jaeschke, K. Altfeld, Proc 22nd World Gas conference, 1-12, Tokyo, June 2003; (no doi available) link: http://www.igu.org/html/wgc2003/WGC_pdffiles/1 0274_1045144488_24432_1.pdf

[2] P. Ulbig, D. Hoburg, Thermochimica Acta 382, 27-35 (2002); doi: 10.1016/S00406031(01)00732-8

[3] J.S: Stufkens, H.J. Bogaard, Analytical Chemistry 47, 383-386 (1975); doi: 10.1021/ac60353a060

[4] S. Udina, M. Carmona, G. Carles, J. Santander, L. Fonseca, S. Marco, Sensors and actuators B: Chemical 2, 551-558 (2008) doi: doi:10.1016/j.snb.2008.05.043

[5] S. Udina, M. Carmona, A. Pardo, C. Calaza, J. Santander, L. Fonseca, S. Marco, Sensors and actuators B: Chemical, accepted for publication Nov 2011 (no doi available yet).

[6] D. Karatzas, A. Chorti, N.M. White, C.J. Harris, IEEE Sensors Journal, 2007, 7, 868-881.

[7] M. Padilla, A. Perera, I. Montoliu, A. Chaudry, K. Persaud, S. Marco, IEEE International Symposium on Intelligent Signal Processing (WISP 2007), 2007, 1-6. 